\title{
New crosslinked cast films based on poly(vinyl alcohol): Preparation and physico-chemical properties
}

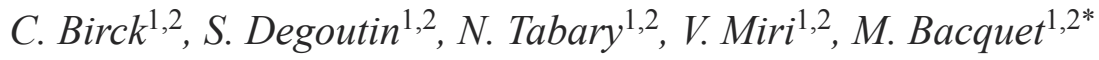 \\ ${ }^{1}$ Unité des Matériaux et Transformations (UMET), CNRS UMR 8207, Equipe ISP, Université Lille 1, 59655 Villeneuve \\ d'Ascq, France \\ ${ }^{2}$ Université Lille Nord de France, 59000 Lille, France
}

Received 14 May 2014; accepted in revised form 13 July 2014

\begin{abstract}
In this paper, we propose a green route to prepare insoluble poly(vinyl alcohol) (PVOH) cast films with potential application as antimicrobial packaging. First PVOH films were cast from different aqueous solutions and analyzed by Differential Scanning Calorimetry (DSC) and Dynamic Mechanical Analysis (DMA) to determine their physical properties under two storage conditions. In order to obtain insoluble films, PVOH was then crosslinked by citric acid (CTR) as confirmed by Nuclear Magnetic Resonance (NMR) analyses. The crosslinking reaction parameters (curing time, crosslinker content) were studied by comparing the characteristics of PVOH/CTR films, such as free COOH content and glass transition temperature $\left(T_{\mathrm{g}}\right)$ value, as well as the impact of the crosslinking reaction on mechanical properties. It was found that for 40 and $10 \mathrm{wt} \%$ CTR contents, 120 and 40 min of crosslinking times were necessary to bind all CTR respectively. Brittle films were obtained for $40 \mathrm{wt} \%$ CTR whereas $10 \mathrm{wt} \%$ CTR content led to ductile films. Finally, films containing hydroxypropyl- $\beta$-cyclodextrin $(\mathrm{HP} \beta \mathrm{CD}$ ), chosen as a potential vector of antimicrobial agent, were prepared. The obtained results show that the incorporation of $\mathrm{HP} \beta \mathrm{CD}$ in the $\mathrm{PVOH}$ matrix does not mainly influence the physical and mechanical properties of the films.
\end{abstract}

Keywords: mechanical properties, poly(vinyl alcohol), cyclodextrin, crosslinking, cast films

\section{Introduction}

Poly(vinyl alcohol) (PVOH) is a non-toxic, biodegradable polymer used in food packaging applications owing to its high barrier properties to oxygen and carbon dioxide $[1,2]$. However, due to its solubility in water, it is often combined with other polymers in multilayer structures, $\mathrm{PVOH}$ being located in the core layer. An alternative route to avoid the use of several polymers would be the crosslinking of PVOH. For this polymer, the most commonly used crosslinkers are dialdehydes, such as glyoxal [3] or glutaraldehyde [4, 5] and polycarboxylic acids. Among the latter, citric acid (CTR) $[6,7]$, succinic acid [8] and tartaric acid [9] have the advantage of being used as food additives. In partic- ular, citric acid (food additive European code E330) is interesting for its low price. It is also well-known as crosslinking agent for compounds bearing hydroxyl groups (like polysaccharides and cyclodextrins) as already reported by our group $[10,11]$.

The aim of this study is to prepare antimicrobial films for food industry, which is emerging in the area of active packaging [12]. In order to confer a prolonged antimicrobial activity to $\mathrm{PVOH}$ crosslinked films, we develop an original approach which consists in adding cyclodextrin in the PVOH matrix. Cyclodextrins present several advantages for our application. These biodegradable cyclic oligosaccharides can be used to form inclusion complexes with antimicrobial agents, in order to release it onto

\footnotetext{
${ }^{*}$ Corresponding author, e-mail: maryse.bacquet@univ-lille1.fr (C) BME-PT
} 
the surface of the food [13-15]. The hydroxypropyl$\beta$-cyclodextrin (HP $\beta C D$ ), selected for this study, is a derivative of the $\beta$-cyclodextrin (food additive $\mathrm{E}$ 459 ) and is non-toxic [16].

The objective of this work is to prepare new films based on PVOH and $\mathrm{HP} \beta \mathrm{CD}$, crosslinked by CTR via a green route. In order to optimize the preparation, different processing parameters such as the PVOH dilution ratio, the formulation of the initial batches with or without cyclodextrin and the crosslinking reaction time are studied. Relationships between processing and physico-chemical properties are investigated using several characterization techniques. The release kinetics of an active principle and the antibacterial activity of the optimized films will be discussed in a following paper.

\section{Experimental procedures}

\subsection{Materials}

Poly (vinyl alcohol) (PVOH, $M_{\mathrm{w}}=31000-$ $50000 \mathrm{~g} \cdot \mathrm{mol}^{-1}, 87-89 \%$ hydrolysis, powder) was purchased from Aldrich Chemicals (St. Quentin Fallavier, France). Hydroxypropyl- $\beta$-cyclodextrin $(\mathrm{HP} \beta \mathrm{CD}$ ) was provided by Roquette (Lestrem, France). This derivative of $\beta$-cyclodextrin $(\beta C D)$ is very soluble in water $(>1000 \mathrm{~g} / \mathrm{L})$. The mean Molar Substitution (MS), which is the average number of hydroxypropyl groups per anhydroglucose unit, is 0.65. Citric acid (CTR), sodium hydroxide and calcium acetate were commercial chemical grade products, supplied by Aldrich Chemicals.

\subsection{Films preparation}

For non-crosslinked PVOH films, PVOH was dissolved in distilled water at three concentrations (1, $5,17 \mathrm{wt} \%$ ) by mechanical agitation with helical blade $(150 \mathrm{rpm})$, in the temperature range $75-90^{\circ} \mathrm{C}$ during 3 to 5 hours. The homogeneous solution was then slowly cooled at room temperature, cast onto a silicone plate and dried under a hood for 16 hours. To study the effect of PVOH dilution, a non-crosslinked $\mathrm{PVOH}$ film was prepared by compression molding at $220^{\circ} \mathrm{C}, 50$ bars, and cooled down to room temperature at $1{ }^{\circ} \mathrm{C} / \mathrm{min}$.

For crosslinked PVOH/CTR films, CTR was added in the homogeneous PVOH solution. The final reagents concentration was equal to $5 \mathrm{wt} \%$. The solution was stirred by mechanical agitation and cast, using similar conditions as previously described. Once the film was formed, the crosslinking reaction was achieved through a heat treatment at $130 \pm 1^{\circ} \mathrm{C}$ during increasing times (from 0 to $960 \mathrm{~min}$ ). Two relative weight compositions of $\mathrm{PVOH} / \mathrm{CTR}$ films were prepared: $90 / 10$ and 60/40.

Crosslinked PVOH/CTR/HP $\beta$ CD films, in weight proportions $80 / 10 / 10$, were prepared following the same protocol as for films without HP $\beta C D$. The proportion of HP $\beta C D$ in the film was fixed considering first an equimolar complex between $\mathrm{HP} \beta C D$ and the later added antimicrobial agent and secondly according to the European directive giving a limit for constituent's migration in food packaging at $10 \mathrm{mg} / \mathrm{dm}^{2}$ [17].

The thickness of each film was $120 \pm 20 \mu \mathrm{m}$.

Two storage conditions have been used for the films. Dried films were obtained by keeping them at $60^{\circ} \mathrm{C}$ during 16 hours under vacuum. Hydrated films were obtained by conditioning at $58 \%$ Relative Humidity (R.H.) during three days (room temperature in an environmental chamber in the presence of $\mathrm{NaBr}$ saturated solutions).

\subsection{Thermogravimetric analysis (TGA)}

TGA was used to determine the water content and the thermal degradation of films. Experiments were performed on a TA Q50 (TA Instruments, United States) apparatus from ambient to $600^{\circ} \mathrm{C}$ with a heating rate of $5^{\circ} \mathrm{C} / \mathrm{min}$. The sample was placed in an open platinum pan under nitrogen.

\subsection{Differential scanning calorimetry (DSC)}

DSC measurements were performed on a TA Q100 (TA Instruments, United States) apparatus under ultra-pure nitrogen at a heating rate of $10^{\circ} \mathrm{C} / \mathrm{min}$. $\mathrm{PVOH}$ and $\mathrm{PVOH} / \mathrm{CTR}$ films (with or without $\mathrm{HP} \beta C D$ ) were analyzed, during the first heating, from -70 to $220^{\circ} \mathrm{C}$ and to $150^{\circ} \mathrm{C}$, respectively. The glass transition temperature $\left(T_{\mathrm{g}}\right)$ was taken at the onset of the heat capacity jump and the melting point $\left(T_{\mathrm{m}}\right)$ at the maximum of the endothermic peak. The crystallinity degree $\left(X_{\mathrm{c}}\right)$ was calculated using the value of $162 \mathrm{~J} / \mathrm{g}$ which is the most cited reference melting enthalpy for PVOH crystals [18-20]. Three replicates were analyzed for each film.

\subsection{X-ray diffraction (XRD)}

Wide-angle-X-ray scattering (WAXS) analysis was performed using a Rigaku rotating anode equipment (Rigaku, Japan) operating at $50 \mathrm{kV}$ and $100 \mathrm{~mA}$. The $\mathrm{Cu} \mathrm{K} \alpha$ radiation $(\lambda=1.54 \AA)$ was selected with a 
point focusing monochromator. The WAXS patterns were recorded on MAR2300 imaging plates. Radial Intensity Profiles, $I(2 \theta)$, were obtained by azimuthal integration of the $2 \mathrm{D}$ patterns.

\subsection{Dynamic mechanical analysis (DMA)}

DMA measurements were performed on a Rheometrics RSA III (TA Instruments, United States) apparatus operating in tensile mode at a frequency of $1 \mathrm{~Hz}$ using a dynamic strain amplitude $\varepsilon_{0}=0.05 \%$ in the temperature range $-50^{\circ} \mathrm{C}<T<150^{\circ} \mathrm{C}$. Specimens with gauge width and length $6 \mathrm{~mm} \times 10 \mathrm{~mm}$ were cut out from the films of PVOH crosslinked or not.

\subsection{Uniaxial tensile measurements}

Uniaxial Tensile testing was conducted on a 4466 Instron (Instron, United Kingdom) machine at room temperature $\left(22^{\circ} \mathrm{C}\right)$ using specimens with 24 and $5 \mathrm{~mm}$ of gauge length and width, respectively. The tensile tests were carried out at a constant crosshead speed of $14.4 \mathrm{~mm} \cdot \mathrm{min}^{-1}$ which corresponds to an initial strain rate of $0.01 \mathrm{~s}^{-1}$. The nominal stress $\sigma_{\mathrm{N}}$ and the nominal strain $\varepsilon_{\mathrm{N}}$ were defined as conventionally by the ratio of the force per the initial crosssection and the elongation per the initial length of the sample, respectively. Three replicates have been analyzed for each film.

\subsection{Nuclear magnetic resonance (NMR)}

${ }^{1} \mathrm{H}$ NMR was used to check the presence of unreacted reagents in films. Each film was immersed in water during 3 days under agitation. Once the film was removed, the solution containing released reagents was freeze-dried. The obtained solid was dissolved in $\mathrm{D}_{2} \mathrm{O}$ and freeze-dried again. The final obtained solid was dissolved in $\mathrm{D}_{2} \mathrm{O}$ and analyzed by ${ }^{1} \mathrm{H}$ NMR using a spectrometer Bruker Avance ${ }^{\mathrm{TM}}$ (Bruker Corporation, Germany) $300 \mathrm{MHz}$ at room temperature.

\subsection{Free $\mathrm{COOH}$ groups quantification}

This quantification was undertaken by Ion Exchange Capacity (IEC) method through $\mathrm{pH}$-metric titration using calcium acetate [21]. Each sample was dried 16 hours at $60^{\circ} \mathrm{C}$ before titration. Typically, $0.1 \mathrm{~g}$ of film sample was stirred in $100 \mathrm{~mL}$ of a calcium acetate solution during 2 hours. The formed acetic acid was titrated by a standard $\mathrm{NaOH}$ solution. The amount of carboxylic functions was calculated following the Equation (1):

$I E C[\mathrm{mmol} / \mathrm{g}]=\frac{C_{\mathrm{b}}[\mathrm{mmol} / \mathrm{mL}] \cdot V_{\mathrm{e}}[\mathrm{mL}]}{\text { sample weight }[\mathrm{g}]}$

where $C_{\mathrm{b}}$ and $V_{\mathrm{e}}$ correspond to the concentration and the equivalent volume of the $\mathrm{NaOH}$ solution respectively. Three replicates were undertaken for each film.

\section{Results and discussion}

A TGA study concerning the thermal behavior of the initial reagents was first carried out (Figure 1). We noticed the lower degradation temperature of CTR $\left(170^{\circ} \mathrm{C}\right)$ compared to the other reagents. Thus, the compression molding method at $220^{\circ} \mathrm{C}$ is not suitable for the preparation of PVOH/CTR films. Therefore, the solvent casting method was chosen as an alternative route for the preparation of our films.

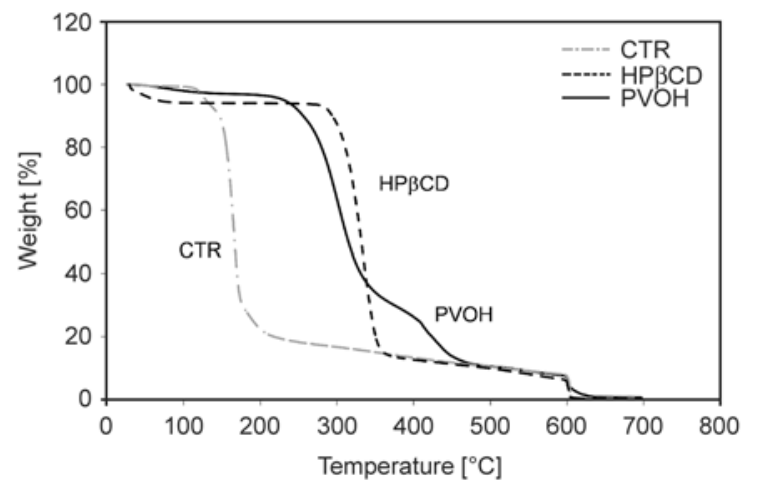

Figure 1. TGA profiles of initial reagents

\subsection{Effect of dilution on the physical properties of non-crosslinked PVOH films}

Polymer dilution is expected to involve a disentanglement of the polymer coils. This phenomenon may induce an increase of crystallinity. This disentanglement may also affect drawability. At high dilution ratio, there is not enough entanglement to ensure a physical crosslinked network behavior, inducing a decrease of the drawability. So a preliminary study was carried out to investigate the effect of dilution on thermal and mechanical properties of $\mathrm{PVOH}$ films, in order to fix the optimal dilution ratio for the further study.

The impact of the dilution ratio during the cast preparation on these thermal transitions is detailed in Table 1. Results for the thermocompression molded film are included for the sake of comparison. 
Table 1. Thermal characteristics of cast and molded films dried or conditioned at 58\% R.H.

\begin{tabular}{|c|c|c|c|c|c|c|c|c|}
\hline \multirow{3}{*}{ 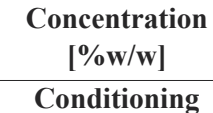 } & \multicolumn{6}{|c|}{ Cast films } & \multirow{2}{*}{\multicolumn{2}{|c|}{ Molded film }} \\
\hline & \multicolumn{2}{|c|}{1} & \multicolumn{2}{|c|}{5} & \multicolumn{2}{|c|}{17} & & \\
\hline & dried & 58\% R.H. & dried & 58\% R.H. & dried & 58\% R.H. & dried & 58\% R.H. \\
\hline wt $\%$ water ${ }^{*}$ & $2 \pm 2$ & $8 \pm 1$ & $1 \pm 1$ & $8 \pm 1$ & $2 \pm 1$ & $7 \pm 2$ & $1 \pm 1$ & $7 \pm 1$ \\
\hline$T_{\mathrm{g}}\left[{ }^{\circ} \mathrm{C}\right]$ & $57 \pm 4$ & $7 \pm 1$ & $57 \pm 1$ & $7 \pm 1$ & $41 \pm 5$ & $6 \pm 1$ & $53 \pm 1$ & $6 \pm 1$ \\
\hline$T_{\mathrm{m}}\left[{ }^{\circ} \mathrm{C}\right]$ & $192 \pm 1$ & $193 \pm 1$ & $192 \pm 1$ & $193 \pm 1$ & $192 \pm 1$ & $193 \pm 1$ & $192 \pm 1$ & $193 \pm 1$ \\
\hline$X_{\mathrm{c}}[\%]^{* *}$ & $26 \pm 1$ & $18 \pm 2$ & $25 \pm 1$ & $16 \pm 1$ & $19 \pm 2$ & $11 \pm 2$ & $14 \pm 1$ & $8 \pm 2$ \\
\hline
\end{tabular}

* water content determined by TGA

${ }^{* *} X_{\mathrm{c}}=$ crystallinity degree

As regards $T_{\mathrm{g}}$ and $T_{\mathrm{m}}$, no major difference is observed whatever the film preparation method. In the dried conditions (corresponding to $2 \pm 1 \mathrm{wt} \%$ of water in the samples), films are characterized by a $T_{\mathrm{g}}$ around $55^{\circ} \mathrm{C}$ and a melting point $T_{\mathrm{m}}$ at $193^{\circ} \mathrm{C}$. For comparison, the reported values for partially hydrolyzed PVOH are $80 \pm 5^{\circ} \mathrm{C}$ for $T_{\mathrm{g}}$ and $185 \pm 5^{\circ} \mathrm{C}$ for $T_{\mathrm{m}}$ [1]. In the present study, these relatively 'low' transition temperatures could be explained by the presence of acetate in this hydrolyzed PVOH (87-89\%). Under $58 \%$ R.H. conditions (corresponding to about $10 \mathrm{wt} \%$ of water), compared to dried one, a $T_{\mathrm{g}}$ drop is observed for all samples, relevant to the plasticizer effect of water molecules which disrupt molecular hydrogen bonds between $\mathrm{PVOH}$ chains in the amorphous phase.

Crystallinity is higher for cast films than for the molded ones, and tends to increase with the dilution ratio. This phenomenon may be attributed to the effect of disentanglements with dilution which promotes crystallization, as expected [22]. With humidity, a significant decrease of $X_{\mathrm{c}}$ is observed for all samples, suggesting that water molecules destroy crystals. This is consistent with results already reported in literature which have shown that water dissolves gradually crystallites by attacking them at the crystalline/amorphous interface [23].

The evolution of the storage modulus, $E^{\prime}$, and loss factor $\tan \delta$, as a function of temperature for molded and PVOH $5 \mathrm{wt} \%$ cast films is presented in Figure 2. In the studied temperature range, all samples exhibit only one relaxation characterized by a pronounced decrease of $E^{\prime}$ and a damping peak of $\tan \delta$. This phenomenon is assigned to the main $\alpha_{\mathrm{a}}$ relaxation associated with the glass transition [24, 25]. As shown in Figure 2, this relaxation is rather independent of film preparation but is very sensitive to water content, in good agreement with DSC results. The peak temperature of $\tan \delta$ decreases from $65^{\circ} \mathrm{C}$ to about $20^{\circ} \mathrm{C}$ for dried and $58 \%$ R.H. films, respectively. Note

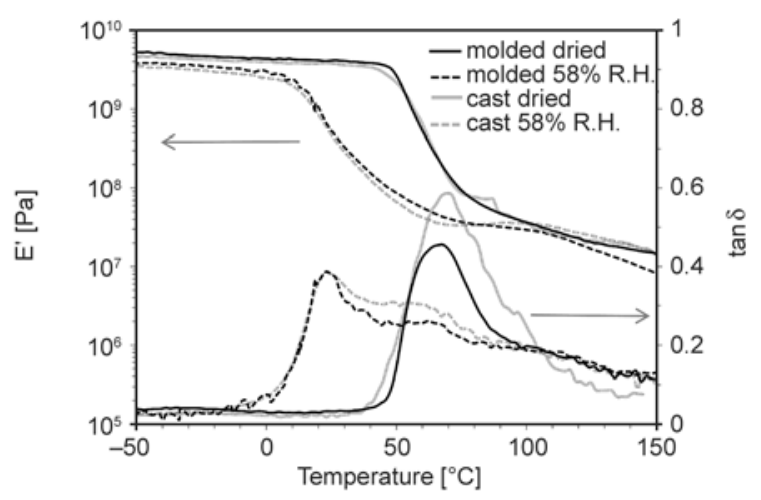

Figure 2. Dynamic mechanical response as a function of temperature of molded and $5 \mathrm{wt} \%$ cast $\mathrm{PVOH}$ films in dried and 58\% R.H. conditions

that the broadness of the peak for humid samples comes from the water evaporation during the experiment.

The yield stress $\sigma_{\mathrm{y}}$ and the strain at break $\varepsilon_{\text {break }}$ values of PVOH films conditioned at 58\% R.H. are reported in Table 2. No result is reported for dried films because, in this case, all samples exhibit a very brittle behavior, related to the drawing temperature well below $T_{\mathrm{g}}$.

All samples display a ductile behavior. No major influence of film preparation on the strain at break is noticed while an increase of $\sigma_{y}$ is observed as initial PVOH concentration increases. One may have expected the reverse evolution considering the variation of the crystal content with the polymer concentration (Table 1). This shows that other structural parameters, than only the crystal content, play an important role in the initiation of plasticity, such as the number of tie molecules.

To sum up, these results do not show any significant difference between all cast films regarding struc-

Table 2. Mechanical data measured for the different films

\begin{tabular}{|l|c|c|c|c|}
\hline $\begin{array}{c}\text { PVOH concentration } \\
{[\% / w / w]}\end{array}$ & \multicolumn{3}{|c|}{ Cast films } & Molded \\
\cline { 2 - 5 } & $\mathbf{1}$ & $\mathbf{5}$ & $\mathbf{1 7}$ & film \\
\hline$\sigma_{\mathrm{y}}[\mathrm{MPa}]$ & $10.3 \pm 0.6$ & $9.1 \pm 0.3$ & $13.8 \pm 1.8$ & $14.7 \pm 1.5$ \\
\hline$\varepsilon_{\text {break }}[\%]$ & $126 \pm 18$ & $140 \pm 16$ & $127 \pm 32$ & $120 \pm 36$ \\
\hline
\end{tabular}


tural and thermomechanical characterizations. These cast films exhibit similar behavior as the molded film. For the following studies, films will be prepared from a total concentration of reactants of $5 \mathrm{wt} \%$, which is the easiest to process in terms of viscosity.

\subsection{Influence of crosslinker amount and crosslinking time on structure of PVOH/CTR films}

The crosslinking reaction involved in this study consists in the esterification between hydroxyl functions of $\mathrm{PVOH}$ and carboxylic functions of CTR as presented in Figure 3a.

The esterification is activated by the preformation of CTR anhydride under the heat treatment. The CTR being a triacid, residual $\mathrm{COOH}$ are present in the three-dimensional network at the end of the reaction. Previous works showed that the esterification occurs from $130^{\circ} \mathrm{C}$ [11].

A constant temperature of $130 \pm 1{ }^{\circ} \mathrm{C}$ was applied for all this study. Two weight proportions of CTR (10 and $40 \mathrm{wt} \%$ ) representative of low and high crosslinker contents were chosen. For both cases, there is an excess of $\mathrm{OH}$ due to $\mathrm{PVOH}$ compared with $\mathrm{COOH}$ functions. Indeed, there are 10 functions $\mathrm{OH}$ for 1 function $\mathrm{COOH}$ for $10 \mathrm{wt} \% \mathrm{CTR}$ films, and 2 functions $\mathrm{OH}$ for 1 function $\mathrm{COOH}$ in $40 \mathrm{wt} \%$ CTR films. The reaction time varied from 0 to $960 \mathrm{~min}$. As shown on Figure 3b, films are transparent. Visual observations showed that the yellowing and the brittleness increase with CTR content and crosslinking time. With $10 \mathrm{wt} \% \mathrm{CTR}$, there is a slight yellowing from $120 \mathrm{~min}$ and films are never brittle. With $40 \mathrm{wt} \%$ CTR, yellowing is observed from $40 \mathrm{~min}$. Films are brittle from $60 \mathrm{~min}$. The yellowing is due to CTR dehydration and formation of a double bond sensitive to oxidation [26].

To check the efficiency of covalent bonding of the different reagents, eventual unreacted CTR and PVOH were obtained by $\mathrm{D}_{2} \mathrm{O}$ extraction of $\mathrm{PVOH} /$ CTR films. The ${ }^{1} \mathrm{H}$ NMR spectra of residual reagents in relation to crosslinking time is presented on Figure 4 (range 4-0 ppm).

The magnitude of the signal for soluble part of the films has been amplified in order to reveal eventual residual compounds and compared to initial reagents. The chemical shifts for PVOH and CTR were attributed to protons also represented on Figure 4.
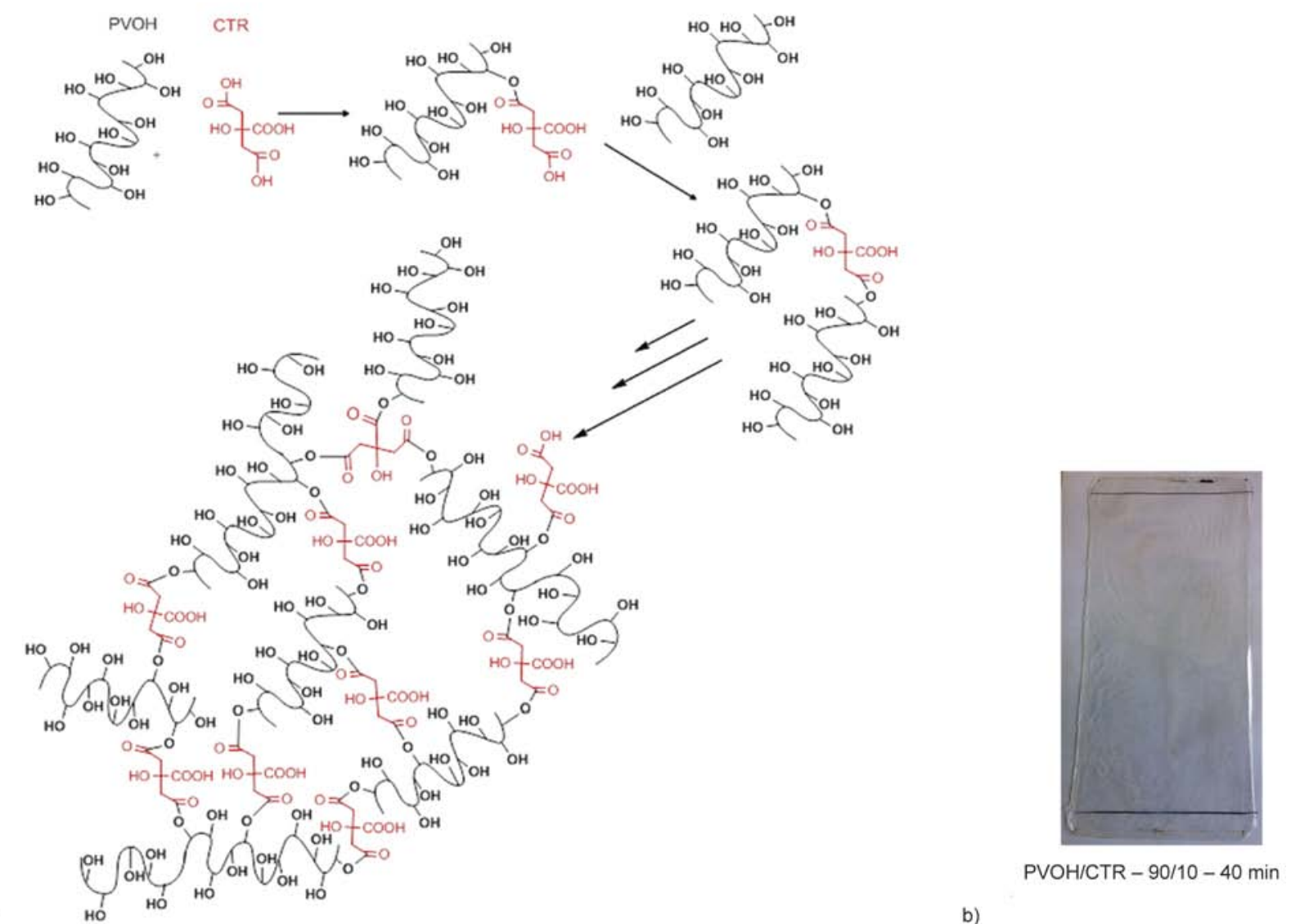

a)

Figure 3. (a) Expected reaction between PVOH and CTR and (b) picture of PVOH/CTR 90/10 film 


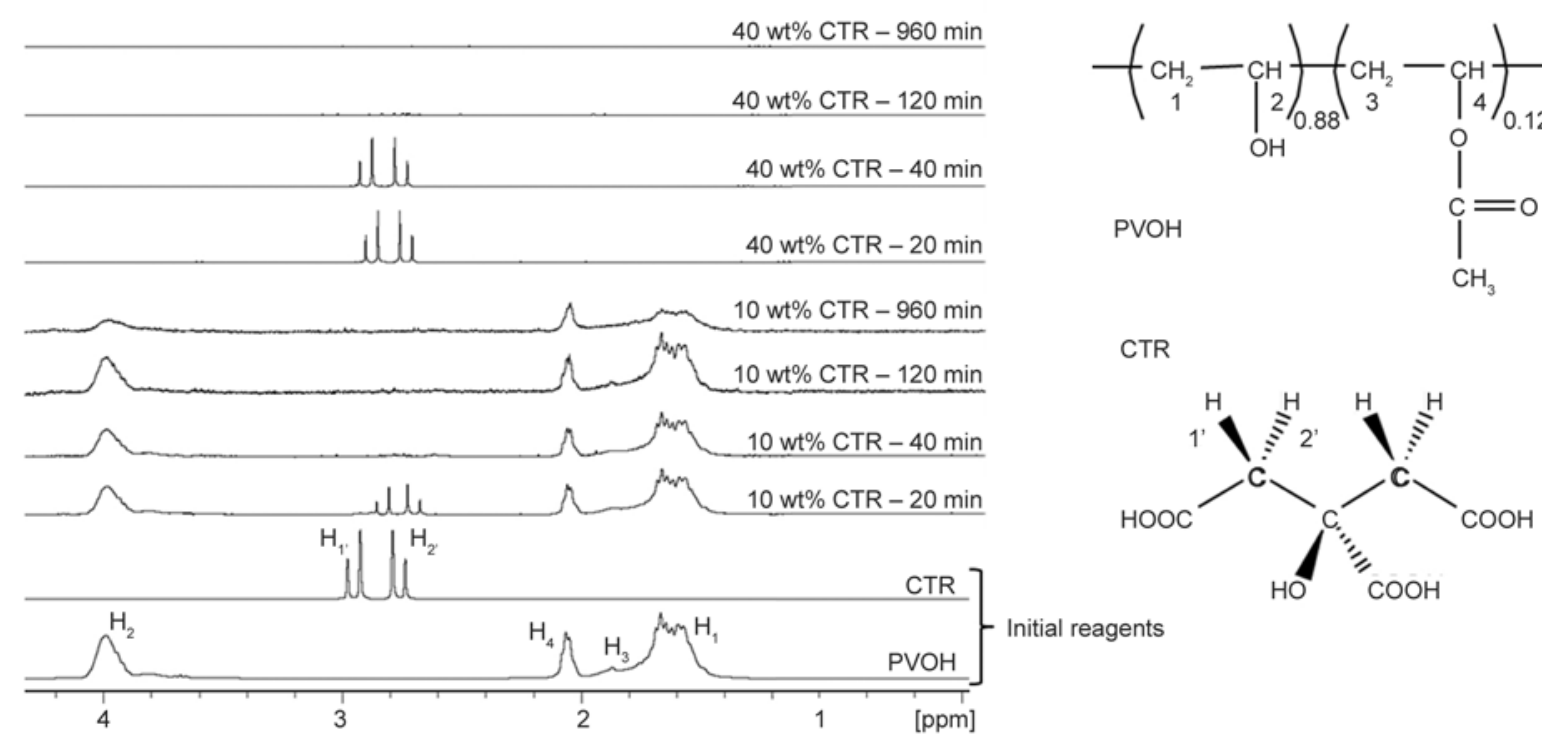

Figure 4. Comparison of ${ }^{1} \mathrm{H}$ NMR spectra for residual reagents in $\mathrm{PVOH} / \mathrm{CTR}$ films related to CTR ratio and reaction time and protons of CTR and $\mathrm{PVOH} 88 \%$

We notice that the quantity of residual compounds progressively decreases with crosslinking time. For $40 \mathrm{wt} \%$ CTR films, PVOH is not detected after $20 \mathrm{~min}$ as well as CTR after $120 \mathrm{~min}$. This means that for this CTR content, a crosslinking time of $120 \mathrm{~min}$ is necessary to bind all CTR to PVOH matrix. For $10 \mathrm{wt} \%$ CTR films, after 40 minutes, CTR is not detected whereas PVOH is still present after $960 \mathrm{~min}$. This indicates that for $10 \mathrm{wt} \% \mathrm{CTR}$, even after 960 min, a weak amount of non crosslinked PVOH chains remains in the films. Nevertheless, the amount of those residual compounds for all the films up to $120 \mathrm{~min}$ is very low (less than $0.1 \%$, determined by weight measurements).

It is well known that the glass transition of the crosslinked polymers depends not only on the main chain rigidity, but also on crosslinking density. In

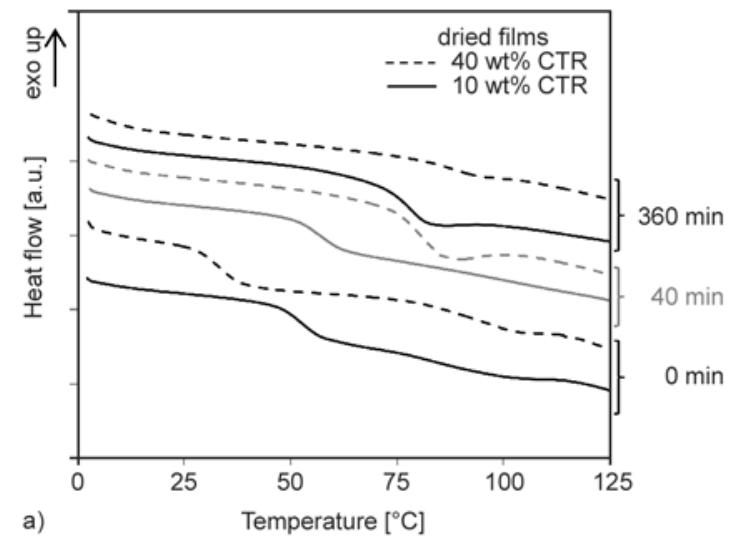

this study, the effect of crosslinking conditions on the PVOH/CTR network is thus investigated considering the evolution of the glass transition. In Figure 5a, typical thermograms for several crosslinking times are shown for both kinds of films. All samples exhibit a heat capacity jump, which is clearly curing time-dependent. The evolution of $T_{\mathrm{g}}$ as a function of crosslinking time is represented on Figure $5 b$ for both storage conditions.

In the case of non crosslinked films (time $=0 \mathrm{~min}$ ), a plasticizer effect of CTR is observed whatever the storage conditions. As expected, this effect is more pronounced for higher CTR concentration. In dried conditions, $\mathrm{PVOH}$ exhibits a $T_{\mathrm{g}}$ at $57^{\circ} \mathrm{C}$, while this latter decreased to $42^{\circ} \mathrm{C}$ and $28^{\circ} \mathrm{C}$ for $\mathrm{PVOH} / \mathrm{CTR}$ 90/10 and PVOH/CTR-60/40 respectively. Similar drop is observed for hydrated non crosslinked films.

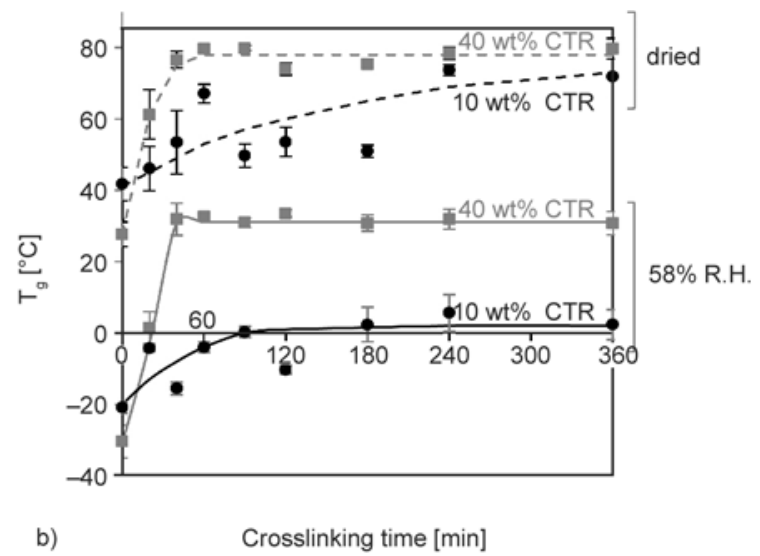

Figure 5. Effect of crosslinking parameters and storage conditions on $T_{\mathrm{g}}$ for different PVOH/CTR films: (a) DSC profiles and (b) $T_{\mathrm{g}}$ as a function of crosslinking time 
Then, the $T_{\mathrm{g}}$ progressively increases with crosslinking time for both proportions of CTR. This increase is slower for $10 \mathrm{wt} \% \mathrm{CTR}$ than for $40 \mathrm{wt} \%$ CTR films. In the latter case, an abrupt rise of $T_{\mathrm{g}}$ is observed followed by a leveling off beyond $60 \mathrm{~min}$ of heating whatever the storage conditions. Considering that $T_{\mathrm{g}}$ increases with crosslink density, this may suggest that no major evolution of the PVOH/ CTR-60/40 network structure occurs above a curing time of $60 \mathrm{~min}$. However, one can observe a decrease of the heat capacity jump $\Delta C_{\mathrm{p}}$ from a crosslinking time of 40 to $360 \mathrm{~min}$. Note that this effect does not occur for PVOH-CTR 90-10. This observation may indicate that the amorphous phase content which relaxes at $T_{\mathrm{g}}$ decreases with crosslinking time for the PVOH/CTR-60/40. Several assumptions could be proposed to explain this result. An increase of crystallinity has been rejected because no ordered phase is evidenced for $40 \mathrm{wt} \%$ CTR whatever the crosslinking time, as it will be shown later. A subsequent crosslinking reaction inducing a restriction of the molecular motions between chemical entanglements is suggested and will be discussed further below.

For dried films, the value of $T_{\mathrm{g}}$ with high crosslinking time (from $240 \mathrm{~min}$ ) is surprisingly quite similar for both CTR concentrations. However, $T_{\mathrm{g}}$ rise for $90 / 10$ films is not so smooth. In fact in the dried state, $T_{\mathrm{g}}$ results from several main combining parameters: the crosslinking density and the hydrogen bonds between hydroxyl groups of PVOH chains but also the intrinsic flexibility of PVOH and CTR chains. As shown in literature, these two parameters acted by a compensation effect between the weakening of the chemical network and the plasticizing effect of the introduced more or less flexible linkers [27]. When films are hydrated and swollen under equilibrium, the decreasing gap of $T_{\mathrm{g}}$ for $10 \mathrm{wt} \%$ CTR is much more pronounced than the one for $40 \mathrm{wt} \%$ CTR. Indeed, the greater proportion of
PVOH hydroxyl groups is, the more they could interact with water molecules resulting in a more pronounced plasticizer effect of water.

The quantification of residual (or free) $\mathrm{COOH}$ groups could also provide some additional information about the formation of the PVOH-CTR network. The esterification reaction, catalyzed by intramolecular CTR anhydride formation (Figure 6) highlighted the origin of these free $\mathrm{COOH}$ groups. If CTR is consumed according to this intramolecular mechanism, namely $2 / 3 \mathrm{COOH}$ functions have reacted. Then, the final theoretical $\mathrm{COOH}$ amount values should be around 0.5 and $1.9 \mathrm{mmol} / \mathrm{g}$ for $\mathrm{PVOH} /$ CTR-90/10 and PVOH/CTR-60/40 films respectively.

The comparison of free $\mathrm{COOH}$ quantification and $T_{\mathrm{g}}$ evolution for the two films is given on Figure 7. As expected, free $\mathrm{COOH}$ functions amount decreases with crosslinking time for both proportions of CTR. A higher decrease is obviously observed for films with $40 \mathrm{wt} \%$ CTR. After 120 minutes, the amount of residual $\mathrm{COOH}$ functions remains constant regardless initial amount of CTR. At this crosslinking time, all CTR is covalently bound as previously proved by NMR (Figure 4). For PVOH/CTR-90/10 (Figure $7 \mathrm{a})$, the experimental value $(0.60 \pm 0.12 \mathrm{mmol} / \mathrm{g})$ is close to the theoretical one $(0.5 \mathrm{mmol} / \mathrm{g})$. Note that the plateau for $T_{\mathrm{g}}$ and residual $\mathrm{COOH}$ are reached for the same crosslinking time (90 $\mathrm{min}$ ). In the case of PVOH/CTR-60/40 (Figure 7b), the behavior is different. First the $\mathrm{COOH}$ experimental value at the plateau $(0.76 \pm 0.13 \mathrm{mmol} / \mathrm{g})$ is lower than the theoretical one $(1.9 \mathrm{mmol} / \mathrm{g})$. Then, the maximum in $T_{\mathrm{g}}$ is achieved from around 40-60 min while at this time $\mathrm{COOH}$ value is still decreasing. In addition, we notice that at $40-60 \mathrm{~min}$, the $\mathrm{COOH}$ value is about $2-3 \mathrm{mmol} / \mathrm{g}$ and close to the calculated one according to the crosslinking mechanism. This can be explained by the fact that for high proportions of CTR, the esterification occurred in two steps. The

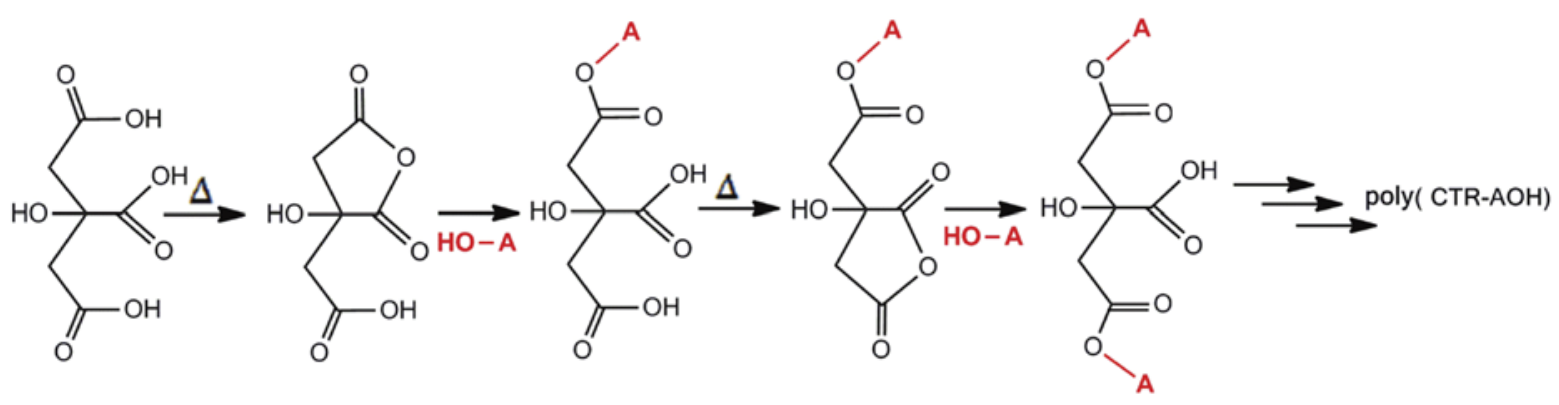

Figure 6. Scheme of intramolecular CTR anhydride formation and esterification between CTR and A-OH $=\mathrm{PVOH}$ 

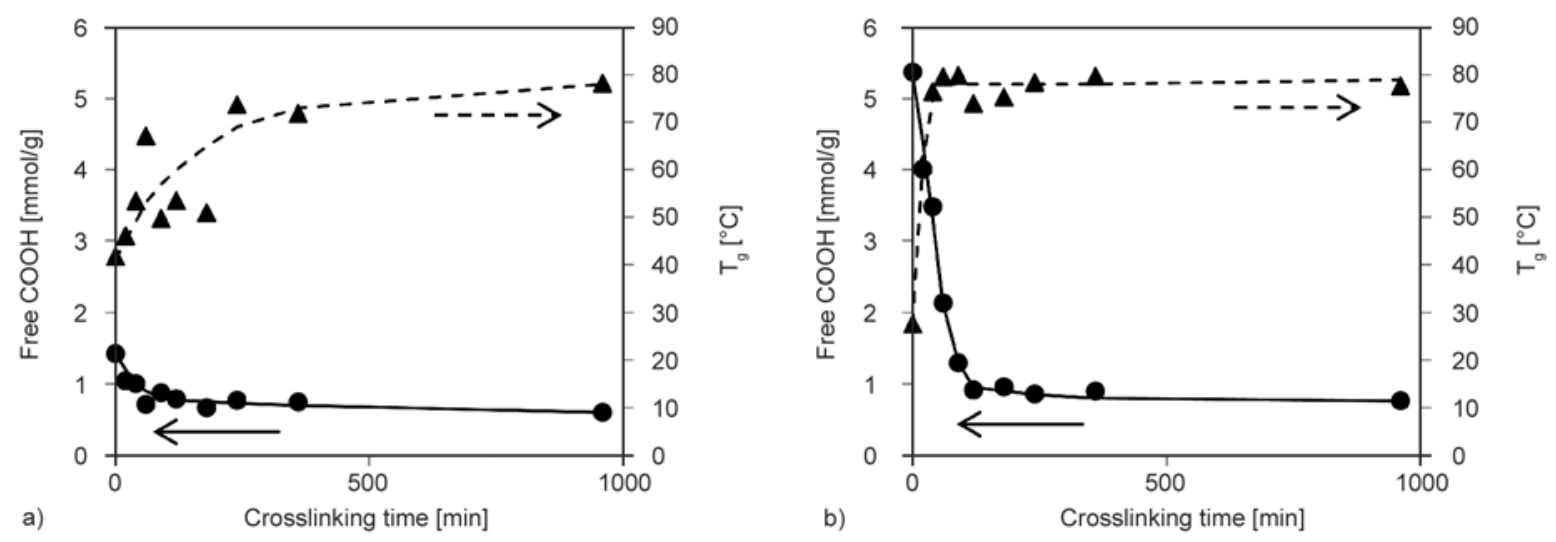

Figure 7. Free $\mathrm{COOH}$ groups and $T_{\mathrm{g}}$ of hydrated $\mathrm{PVOH} / \mathrm{CTR}$ films as a function of crosslinking time for (a) PVOH/CTR 90/10 and (b) PVOH/CTR 60/40

first one may correspond to the grafting of CTR on PVOH chains. Then the spatial proximity between $\mathrm{COOH}$ functions already grafted allows the formation of anhydrides via an intermolecular mechanism and therefore additional post-esterification reaction involving chemical groups close to previous crosslinking points. This phenomenon has no major effect on $T_{\mathrm{g}}$ value neither on the global crosslinking degree but may result in a decrease of the heat capacity jump at $T_{\mathrm{g}}$ as previously mentioned.

\subsection{Physical and mechanical characterization of PVOH/CTR films}

It is interesting to check if some crystallinity is present in initial PVOH/CTR films and remains after crosslinking reaction. PVOH/CTR films exhibit a degradation phenomenon well below the melting point of PVOH $\left(T_{\mathrm{m}}=185 \pm 5^{\circ} \mathrm{C}\right)$. Therefore, DSC cannot be used to highlight any melting peak in this case. So, X-ray diffractograms of PVOH/CTR films were recorded as a function of crosslinking time (Figure 8). Whatever the CTR content and crosslink-

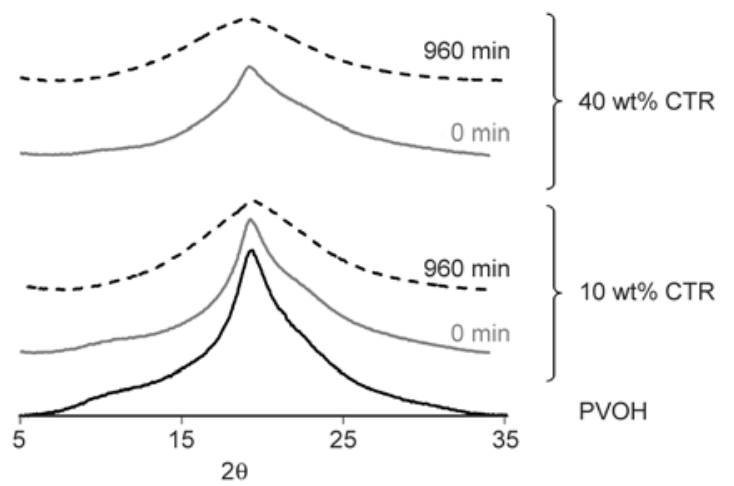

Figure 8. WAXS patterns of hydrated PVOH/CTR films for different crosslinking times ing time, only one broad diffraction located at around $2 \theta=20^{\circ}$ is observed without any evidence of crystalline peak which suggests that all samples are mainly in disordered state.

In Figure 9, typical DMA curves of three hydrated PVOH/CTR-90/10 films are shown: non crosslinked one and two crosslinked ones. An increase of the main relaxation temperatures with crosslinking time is observed, in good agreement with previous DSC results. As expected, the storage modulus $E^{\prime}$ increases with the heating time, below as well as above $T_{\mathrm{g}}$, relevant to an increase of the crosslink density which rigidifies the polymer network. Additionally, one can notice the presence of two peaks in the $\tan \delta$ curves indicating various relaxations in the amorphous phase, even in the non crosslinked sample. This could suggest that the concentration of CTR is not homogeneous at local scale inside the sample.

The effect of crosslinking conditions on tensile behavior is illustrated on Figure 10. For the noncrosslinked films ( $t=0 \mathrm{~min})$, one can observe the

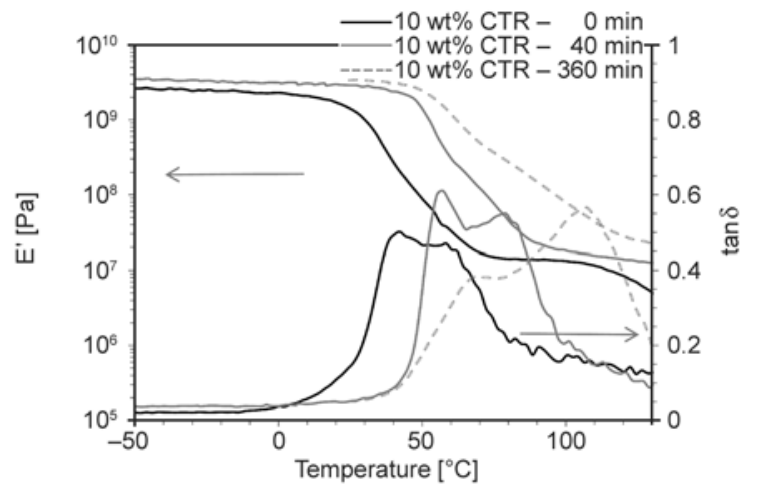

Figure 9. Dynamic mechanical response as a function of temperature of hydrated PVOH/CTR 90/10 films for different crosslinking times 


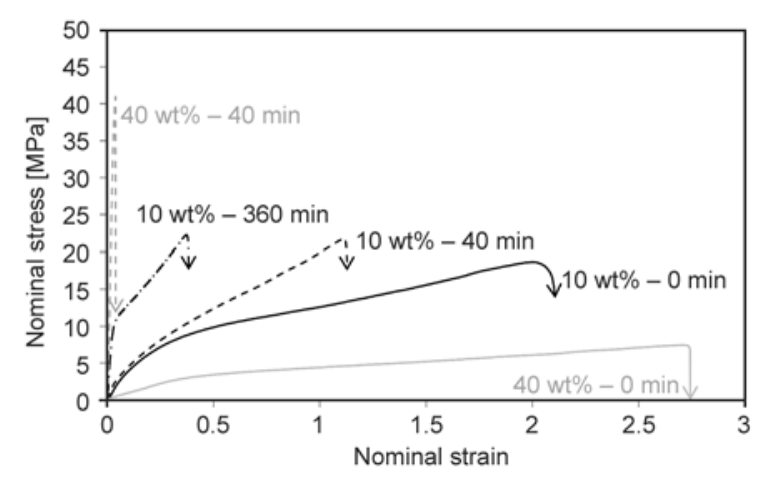

Figure 10. Nominal stress-strain curves of the PVOH/CTR films for different proportions of CTR and crosslinking times under uniaxial drawing at $T=22^{\circ} \mathrm{C}$ at $58 \%$ R.H.

plasticizer effect of CTR indicated by an improvement of ductility as CTR concentration is increased. PVOH/CTR-60/40 samples exhibit a brittle behavior from $40 \mathrm{~min}$ curing, related to the highly crosslinking density preventing any plastic deformation. Owing to their brittleness, we decided to focus our attention only on the films containing $10 \%$ CTR.

All samples exhibit a homogeneous deformation. The stress level increases and the strain at break significantly decreases as the crosslinking time is increased. This behavior is related to the reduction of the chemical entanglement mass involving an enhancement of the rigidity and reduction of the limit of extensibility of chain segments between entanglements.

\subsection{Influence of HPßCD on films properties with $10 \%$ of CTR}

One of the main objectives of this study is to incorporate HP $\beta C D$ in the polymer matrix. This cyclodextrin derivative would be used as a vector for prolonged release of a food preservative. As $\mathrm{PVOH}$, $\mathrm{HP} \beta \mathrm{CD}$ possesses $\mathrm{OH}$ functions which may react with $\mathrm{COOH}$ functions of CTR, allowing it to take part of the three-dimensional network. Because films with $40 \mathrm{wt} \%$ CTR are too brittle from $40 \mathrm{~min}$ of crosslinking, only films containing $10 \mathrm{wt} \%$ CTR are studied. Thus, the influence of the HP $\beta C D$ addition on film properties was investigated on films containing $80 \mathrm{wt} \% \mathrm{PVOH}, 10 \mathrm{wt} \% \mathrm{HP} \beta \mathrm{CD}$ and $10 \mathrm{wt} \%$ CTR using similar approach as previously. In that case, $\mathrm{PVOH} / \mathrm{HP} \beta \mathrm{CD} / \mathrm{CTR}-80 / 10 / 10$ and $\mathrm{PVOH} /$ CTR-90/10 cast solutions contain similar quantities of $\mathrm{OH}$ groups per gram.
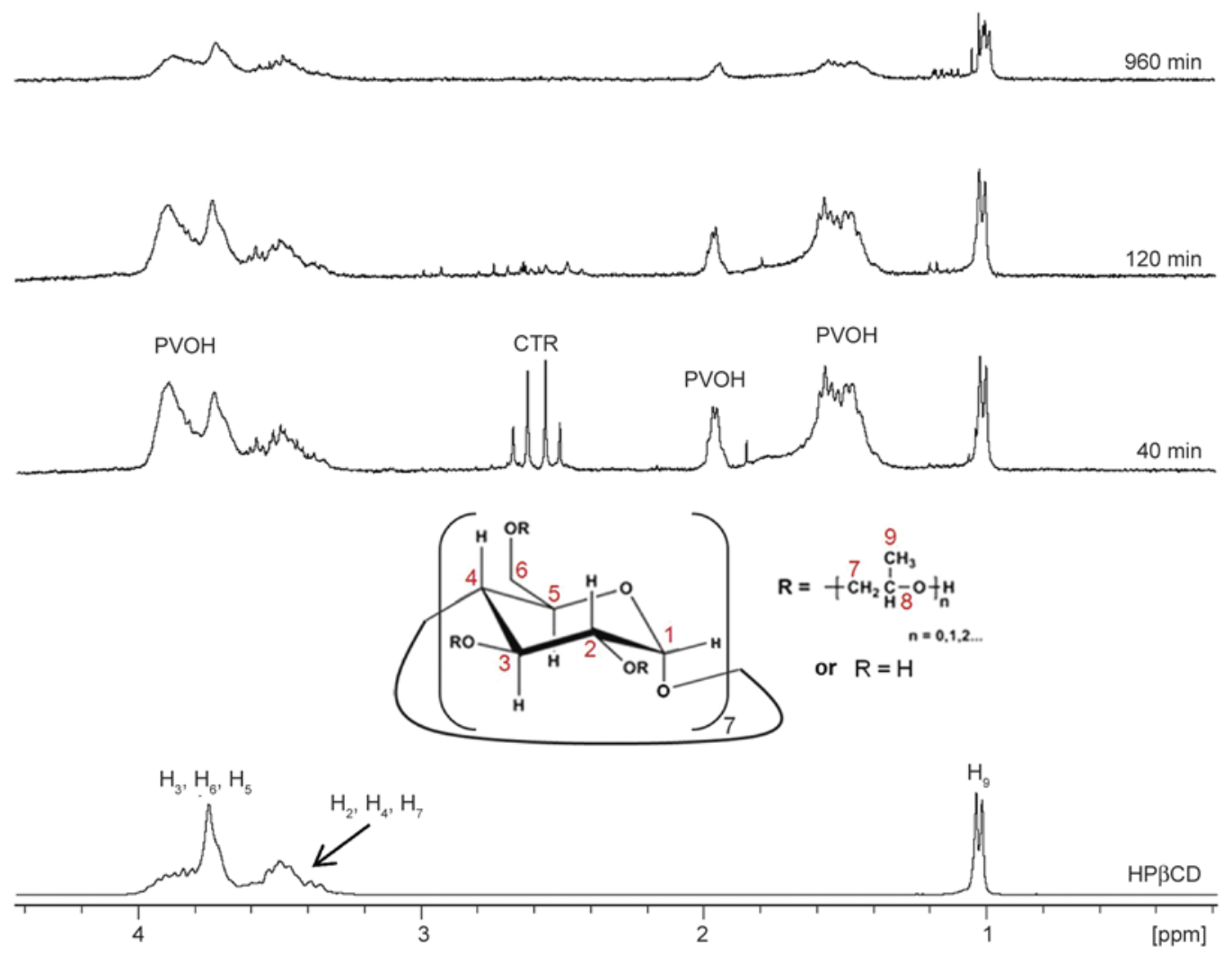

Figure 11. ${ }^{1} \mathrm{H}$ NMR spectra in $\mathrm{D}_{2} \mathrm{O}$ of residual reagents of PVOH/CTR/HP $\beta C D$ films with $10 \mathrm{wt} \% \mathrm{CTR}$ and $10 \mathrm{wt} \%$ $\mathrm{HP} \beta \mathrm{CD}$ for three crosslinking times 
The ${ }^{1} \mathrm{H}$ NMR spectra of unreacted reagents obtained by solvent extraction for $\mathrm{PVOH} / \mathrm{HP} \beta \mathrm{CD} / \mathrm{CTR}$ crosslinked films is presented on Figure 11. As previously, the intensity of the spectra has been magnified to reveal the low amounts of residual compounds. As observed for PVOH/CTR films (Figure 4), CTR is completely bound at $120 \mathrm{~min}$, whereas $\mathrm{PVOH}$ and HP $\beta \mathrm{CD}$ are not completely bound even after $960 \mathrm{~min}$. However the amounts of residual reagents are very low $(<0.1 \%$, determined by weight measurements).

The kinetics of the crosslinking reaction is evaluated following the free $\mathrm{COOH}$ content as a function of crosslinking time (Figure 12). Results show the same behavior as for PVOH/CTR films with $10 \mathrm{wt} \%$ CTR (Figure 7). $\mathrm{COOH}$ functions amount decreases with crosslinking time and reaches a quasi-plateau. The final amount of the free $\mathrm{COOH}$ groups is the same with or without $\mathrm{HP} \beta \mathrm{CD}$. The concentration of $10 \mathrm{wt} \%$ HP $\beta C D$ does not modify the crosslinking kinetics.

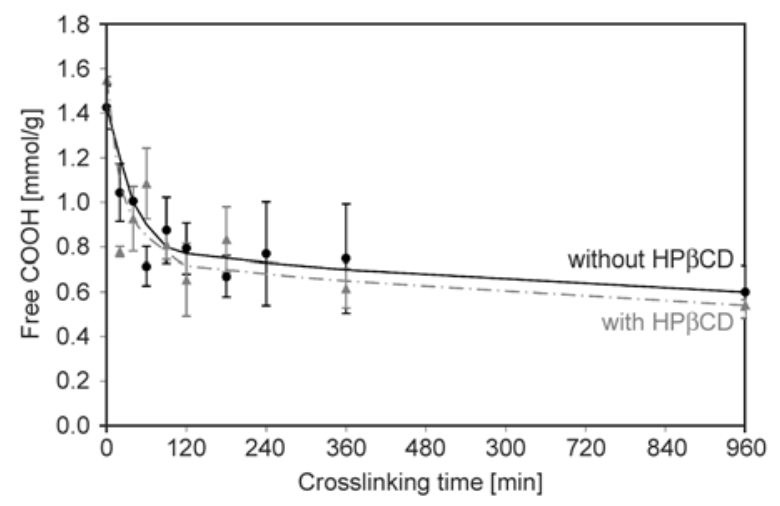

Figure 12. Free $\mathrm{COOH}$ quantification of $\mathrm{PVOH} / \mathrm{CTR}$ and $\mathrm{PVOH} / \mathrm{HP} \beta \mathrm{CD} / \mathrm{CTR}$ films with $10 \mathrm{wt} \% \mathrm{CTR}$ as a function of crosslinking time

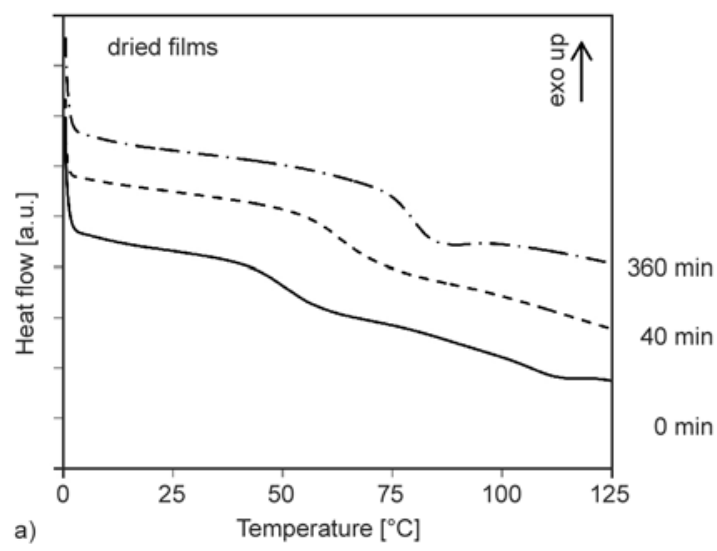

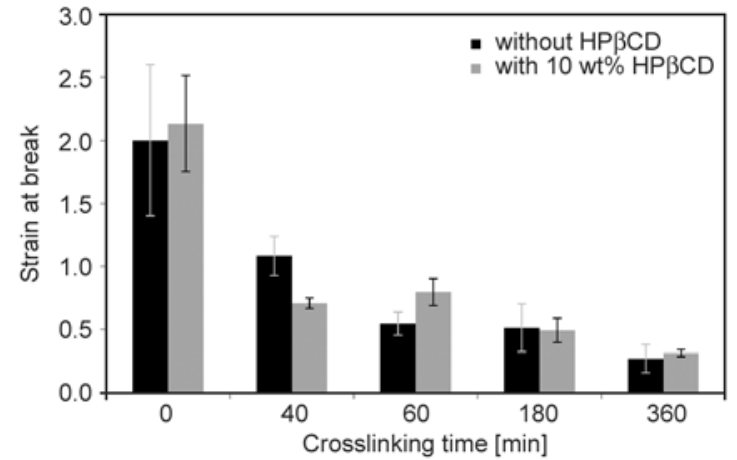

Figure 14. Strain at break at $22^{\circ} \mathrm{C}$ of $\mathrm{PVOH} / \mathrm{CTR}$ and $\mathrm{PVOH} / \mathrm{HP} \beta \mathrm{CD} / \mathrm{CTR}$ films, conditioned at $58 \%$ R.H., as a function of crosslinking time

It is also important to study the effect of $\mathrm{HP} \beta C D$ on film flexibility through $T_{\mathrm{g}}$ analysis (Figure 13). We notice no plasticizer effect of HP $\beta C D$ on non-crosslinked films ( $t=0 \mathrm{~min})$. The incorporation of $\mathrm{HP} \beta \mathrm{CD}$ in the polymer network seems to rigidify it. $\mathrm{HP} \beta C D$ is a bulky and rigid molecule. Its presence should decrease the global mobility of polymer chains.

Finally, drawing experiments were carried out and values of strain at break are reported on Figure 14. For all crosslinking times, the presence of $\mathrm{HP} \beta \mathrm{CD}$ does not affect significantly the drawability of films. All samples present a ductile behavior.

\section{Conclusions}

Films based on PVOH and crosslinked with CTR, have been prepared in two steps by solvent casting method followed by heat treatment. Different characteristics of the films (chemical structure, $T_{\mathrm{g}}$, crystallinity and tensile properties) were studied in relation to their formulation and the crosslinking time. Films with high content of CTR $(40 \mathrm{wt} \%)$ are too brittle for the considered food application while films

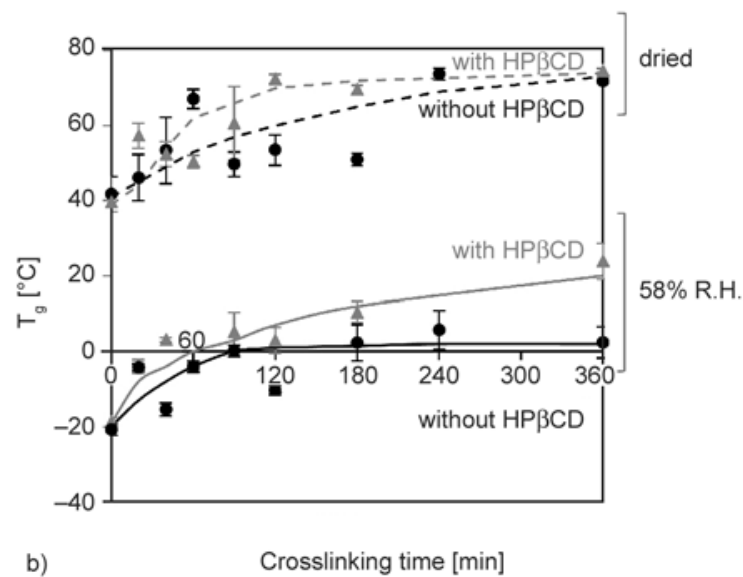

Figure 13. (a) DSC profiles of $\mathrm{PVOH} / \mathrm{HP} \beta \mathrm{CD} / \mathrm{CTR}$ films and (b) values of $T_{\mathrm{g}}$ as a function of crosslinking time with or without $\mathrm{HP} \beta C D$ 
with low content of CTR (10 wt\%) are transparent and show a ductile behavior. However, we highlighted that even after 960 min of crosslinking, a weak quantity of PVOH is not bound. We could expect that an intermediate formulation of $\mathrm{PVOH}$ and CTR could be more suitable for the targeted application. Moreover, during the crosslinking reaction, a part of $\mathrm{COOH}$ functions remains free. We could take advantage of these acid functions for further food storage. Finally, the incorporation of $10 \mathrm{wt} \%$ of cyclodextrin derivative as food additive carrier does not influence the characteristics of the films containing $10 \mathrm{wt} \% \mathrm{CTR}$. This formulation will be used for further studies.

\section{References}

[1] Finch C. A.: Polyvinyl alcohol development. Wiley, New York (1992).

[2] Imam S. H., Cinelli P., Gordon S. H., Chiellini E.: Characterization of biodegradable composite films prepared from blends of poly(vinyl alcohol), cornstarch, and lignocellulosic fiber. Journal of Polymers and the Environment, 13, 47-55 (2005).

DOI: $10.1007 / \mathrm{s} 10924-004-1215-6$

[3] Conte A., Buonocore G. G., Sinigaglia M., Del Nobile M. A.: Development of immobilized lysozyme based active film. Journal of Food Engineering, 78, 741-745 (2007).

DOI: $10.1016 /$ j.jfoodeng.2005.11.013

[4] Hasimi A., Stavropoulou A., Papadokostaki K. G., Sanopoulou M.: Transport of water in polyvinyl alcohol films: Effect of thermal treatment and chemical crosslinking. European Polymer Journal, 44, 40984107 (2008).

DOI: $10.1016 / j$.eurpolymj.2008.09.011

[5] Mansur H. S., Sadahira C. M., Souza A. N., Mansur A. A. P.: FTIR spectroscopy characterization of poly (vinyl alcohol) hydrogel with different hydrolysis degree and chemically crosslinked with glutaraldehyde. Materials Science and Engineering: C, 28, 539-548 (2008). DOI: $10.1016 / \mathrm{j} . \mathrm{msec} .2007 .10 .088$

[6] Shi R., Bi J., Zhang Z., Zhu A., Chen D., Zhou X., Zhang L., Tian W.: The effect of citric acid on the structural properties and cytotoxicity of the polyvinyl alcohol/starch films when molding at high temperature. Carbohydrate Polymers, 74, 763-770 (2008).

DOI: 10.1016/j.carbpol.2008.04.045

[7] Wang S., Ren J., Li W., Sun R., Liu S.: Properties of polyvinyl alcohol/xylan composite films with citric acid. Carbohydrate Polymers, 103, 94-99 (2014). DOI: $\underline{10.1016 / \text { j.carbpol.2013.12.030 }}$
[8] Ajji Z.: Preparation of poly(vinyl alcohol) hydrogels containing citric or succinic acid using gamma radiation. Radiation Physics and Chemistry, 74, 36-41 (2005).

DOI: 10.1016/j.radphyschem.2004.12.005

[9] Salt Y., Hasanoğlu A., Salt İ., Keleşer S., Özkan S., Dinçer S.: Pervaporation separation of ethylacetatewater mixtures through a crosslinked poly(vinylalcohol) membrane. Vacuum, 79, 215-220 (2005).

DOI: 10.1016/j.vacuum.2005.03.010

[10] Martel B., Morcellet M., Ruffin D., Ducoroy L., Weltrowski M.: Finishing of polyester fabrics with cyclodextrins and polycarboxylic acids as crosslinking agents. Journal of Inclusion Phenomena and Macrocyclic Chemistry, 44, 443-446 (2002). DOI: 10.1023/A:1023080221850

[11] Martel B., Ruffin D., Weltrowski M., Lekchiri Y., Morcellet M.: Water-soluble polymers and gels from the polycondensation between cyclodextrins and poly (carboxylic acid)s: A study of the preparation parameters. Journal of Applied Polymer Science, 97, 433-442 (2005).

DOI: 10.1002/app.21391

[12] Dainelli D., Gontard N., Spyropoulos D., Zondervanvan den Beuken E., Tobback P.: Active and intelligent food packaging: Legal aspects and safety concerns. Trends in Food Science and Technology, 19, S103S112 (2008).

DOI: $10.1016 / \mathrm{j}$. tifs.2008.09.011

[13] Teixeira B. N., Ozdemir N., Hill L. E., Gomes C. L.: Synthesis and characterization of nano-encapsulated black pepper oleoresin using hydroxypropyl betacyclodextrin for antioxidant and antimicrobial applications. Journal of Food Science, 78, 1913-1920 (2013). DOI: $10.1111 / 1750-3841.12312$

[14] Piercey M. J., Mazzanti G., Budge S. M., Delaquis P. J., Paulson A. T., Truelstrup Hansen L.: Antimicrobial activity of cyclodextrin entrapped allyl isothiocyanate in a model system and packaged fresh-cut onions. Food Microbiology, 30, 213-218 (2012).

DOI: $10.1016 / \mathrm{j} . \mathrm{fm} .2011 .10 .015$

[15] Astray G., Gonzalez-Barreiro C., Mejuto J. C., RialOtero R., Simal-Gándara J.: A review on the use of cyclodextrins in foods. Food Hydrocolloids, 23, 16311640 (2009).

DOI: $10.1016 /$ j.foodhyd.2009.01.001

[16] Frömming K-H., Szejtli J.: Cyclodextrins in pharmacy. Springer, Dordrecht (1994).

[17] Council Direktrive 85/572/EEC D. Laying down the list of simulants to be used for testing migration of constituents of plastic materials and articles intended to come into contact with foodstuffs (1985).

[18] Fukae R., Yamamoto T., Fujita Y., Kawatsuki N., Sangen O., Kamachi M.: Poly(vinyl alcohol) with high diad-syndiotacticity and high melting point. Polymer Journal, 29, 293-295 (1997).

DOI: $10.1295 /$ polymj.29.293 
[19] Wunderlich B.: Macromolecular Physics, Volume 1: Crystal structure, morphology, defects. Academic Press, New York (1973).

[20] Tubbs R. K.: Melting point and heat of fusion of poly (vinyl alcohol). Journal of Polymer Science Part A: General Papers, 3, 4181-4189 (1965).

DOI: 10.1002/pol.1965.100031213

[21] Ducoroy L., Martel B., Bacquet M., Morcellet M.: Cation exchange finishing of nonwoven polyester with polycarboxylic acids and cyclodextrins. Journal of Applied Polymer Science, 103, 3730-3738 (2007). DOI: 10.1002/app.25249

[22] Darras O., Seguela R., Rietsch F.: Dried gels from linear low-density polyethylene: Morphology, thermal behavior, and mechanical properties. Journal of Polymer Science Part B: Polymer Physics, 30, 349-359 (1992).

DOI: $\underline{10.1002 / \text { polb.1992.090300405 }}$
[23] Hodge R. M., Edward G. H., Simon G. P.: Water absorption and states of water in semicrystalline poly(vinyl alcohol) films. Polymer, 37, 1371-1376 (1996).

DOI: 10.1016/0032-3861(96)81134-7

[24] Hoffman J. D.: Anelastic and dielectric effects in polymeric solids. Wiley, New York (1969).

[25] Nishio Y., Manley R. S. J.: Cellulose-poly(vinyl alcohol) blends prepared from solutions in N,N-dimethylacetamide-lithium chloride. Macromolecules, 21, 1270-1277 (1988). DOI: $10.1021 / \mathrm{ma} 00183 \mathrm{a} 016$

[26] Lu Y., Yang C. Q.: Fabric yellowing caused by citric acid as a crosslinking agent for cotton. Textile Research Journal, 69, 685-690 (1999). DOI: $10.1177 / 004051759906900909$

[27] Krumova M., López D., Benavente R., Mijangos C., Pereña J. M.: Effect of crosslinking on the mechanical and thermal properties of poly(vinyl alcohol). Polymer, 41, 9265-9272 (2000). DOI: 10.1016/S0032-3861(00)00287-1 